\title{
A Research on the Quality Features of Ice Cream Produced Using Some Fat Substitutes
}

\author{
Abdulkerim Hatipoğlu ${ }^{1}$ and Hüseyin Türkoğlu ${ }^{2}$ \\ 1. Department of Nutrition and Dietetics, Faculty of Health Sciences, Mardin Artuklu University, Mardin 47200, Turkey \\ 2. Department of Food Processing, Ula Ali Koçman Vocational High School, Muğla Sitkı Koçman University, Muğla 48640, Turkey
}

\begin{abstract}
The aim of this study was to determine some quality properties of fat-reduced ice creams produced using some fat substitutes: $10 \%$ fat ice cream was considered as the control group; $6 \%$ Simplesse ${ }^{\circledR} 100$ or $2 \%$ Maltrin040 was added as fat substitute to $7.5 \%, 5 \%$ and $2.5 \%$ fat ice cream. The organoleptic (firmness, melting resistance, structure, gumminess, melting in mouth, feeling in mouth, icy structure, smoothness, flavor, cream taste, milk powder taste, foreign taste, vanilla taste and general acceptability), physical (viscosity, overrun, melting rate and first drip time) and chemical (dry matter, fat, fat in dry matter, protein, $\mathrm{pH}$, titration acidity and ash) properties of ice cream samples were investigated on the 1st, 7th, 15th, 30th, 60th and 90th days of the storage. According to results of the research, the viscosity of $7.5 \%$ fat ice cream Maltrin040 or Simplesse ${ }^{\circledR} 100$ added was higher than the control group. Moreover, decrease in fat adversely affected organoleptic properties of the ice cream. As a result of the findings, $7.5 \%$ fat ice creams $6 \%$ Simplesse ${ }^{\circledR} 100$ added can be recommended for ice creams to be produced by reducing fat ratio. In the near future, the production of ice-creams with fat substitutes at an industrial level will be of great importance for low-energy and vegetable fat dieters.
\end{abstract}

Key words: Ice cream, fat substitute, simplesse, maltrin.

\section{Introduction}

Today, the demand for low calorie products is increasing for many different reasons such as nutrition, health and weight control. Therefore, special food formulations are being developed for conditions such as diabetes caused by excessive energy intake, nutritional diseases or insufficient dietary fiber consumption $[1,2]$.

In this context, many fat substitutes are also used in ice cream formulations. Fat substitutes in question can be used to stabilize ice cream, provide emulsion, slow down melting, improve texture, provide overrun, and fix the sensation left in the mouth without impairing the sensory properties of the ice cream. Thus, the unwanted properties resulting from decreasing the fat ratio or removing the fat in the final product can be minimized [3, 4]. The fat used in ice cream is an

Corresponding author: Abdulkerim Hatipoğlu, assistant professor, research field: advanced dairy processing. important component that ensures the structure and texture to be smooth, gives the ice cream taste-aroma richness, also improves the rheological properties of the ice cream and contributes to the melting resistance of ice cream [5]. Fat substitutes are substances that can mimic the physical and sensory properties of some foods but provide significantly less calories [6]. These substances are generally divided into three groups according to their composition: lipid, protein and carbohydrate based. Each group has different functional properties and can be used alone or as a mixture [7, 8]. It consists of carbohydrate-based fat substitutes, modified starch species, maltodextrins from various sources, cellulose derivatives (microcrystalline cellulose, methyl cellulose and hydroxypropyl methyl cellulose), inulin, pectin, polydextrose and other dietary fibers. Protein-based fat substitutes are generally produced from concentrated whey proteins [9]. Lipid-based oil substitutes consist of emulsifiers, medium chain 
triacylglycerols or surface-active structural lipids that can stabilize emulsions [7].

In this study, the possibilities of the use of some fat substitutes in different ratios were investigated in order to reduce the fat ratio without causing a negative change in the sensory properties of ice cream.

\section{Materials and Methods}

\subsection{Materials}

Cow milk ( $3 \%$ fat), milk powder and cream used in the production of ice cream (Pınar Milk and Dairy Products Corporation) were purchased from the market. Simplesse ${ }^{\circledR} 100$ and Maltrin040 used as fat substitutes were supplied from CP Kelco Co. (Teknorama Co., İstanbul). The sugar used (sucrose) and vanilla were obtained from the market. GMS used as emulsifier, Karragenan and Guar Gum used as stabilizer were supplied from Incom Industrial Machinery Import and Export Co. (Mersin).

\subsection{Methods}

\subsubsection{Preparation of the Ice-Cream Mixes}

In the study, the fat-free dry matter of the mixes was adjusted with milk powder: $10 \%$ fat ice-cream was accepted as the control group and the fat ratios of the other ice-creams were adjusted with the addition of cream to be $2.5 \%, 5 \%$ and $7.5 \%$. The ice cream mixes were prepared by adding $18 \%$ crystallized sugar, $0.1 \%$ guar gum as stabilizer, $0.25 \%$ carrageenan and $0.1 \%$ glycerol monostearate (GMS) as emulsifier and $0.04 \%$ vanilla as flavorant. While $2 \%$ Maltrin040 was added to a portion of the fat-reduced ice cream mixes, $6 \%$ Simplesse ${ }^{\circledR} 100$ was added to parallel production mixes.

After the main components of the ice cream mix were weighed and prepared, the milk started to be heated. When the mixture reached $40{ }^{\circ} \mathrm{C}$, cream, milk powder and some of the sugar were added and mixed. When the temperature reached $50-55{ }^{\circ} \mathrm{C}$, the remaining sugar, GMS, guar gum, carrageenan, fat substitute and vanilla were mixed well and added to the main mixture. When the temperature of the mixture reached $85{ }^{\circ} \mathrm{C}$, it was slowly mixed and pasteurized at this temperature for $10 \mathrm{~min}$. Then the ice cream mix was cooled and put in plastic boxes and matured in cold storage at $4 \pm 1{ }^{\circ} \mathrm{C}$ for $18 \mathrm{~h}$.

\subsubsection{Ice Cream Production}

After resting, the mixes were turned into ice cream in a batch type ice cream machine (Uğur brand) at Şanlıurfa Vocational High School Laboratory of Harran University. The freezing process lasted $10 \mathrm{~min}$ for each portion. Fat and dry matter ratios of control group ice creams were adjusted by adding cream instead of fat substitute. First, control group ice creams were produced and then the fat-substituted ones. And $0.04 \%$ vanilla flavor was added to the ice cream. The ice creams from the ice cream machine were placed in plastic containers for sensory, physical and chemical analysis and stored at $-28 \pm 2{ }^{\circ} \mathrm{C}$ for 90 days.

\subsubsection{Chemical Analyses}

Chemical analyses of ice cream samples were performed on the 1st, 7th, 15th, 30th, 60th and 90th days of storage. Dry matter amount (\%) was determined by gravimetric method, fat amount (\%) was determined by Gerber method, total protein (\%) was determined by micro-Kjeldahl method [10, 11]. In addition, $\mathrm{pH}$ and titration acidity was determined [12].

\subsubsection{Physical Analyses}

Physical analyses were performed on the last day of storage (90th day). The viscosities (cP) of the mixes were determined by measuring the viscosity meter (Poulten Selge Elee, Wickford, Essex, UK) at 6 and 20 and $50 \mathrm{rpm}$. In addition, the overrun (\%) of ice cream samples [12], melting rate (\%) [13] and the first drip time [13] were determined.

\subsubsection{Organoleptic Analyses}

Organoleptic analyses of the ice cream samples were performed on the 1st, 7th, 15th, 30th, 60th and 90th days of storage. The organoleptic evaluation of the experiment ice-cream was made by a group of 8 panelists composed of lecturers of Harran University 
Faculty of Engineering Department of Food Engineering using the hedonic type scale (9: best, 1: worst score) prepared by us considering the criteria given by Gürsel and Karacabey [12], Roland et al. [14], Chung et al. [15] and Atsan [16]. The effect of the fat substitutes added in the organoleptic evaluation on the firmness, resistance to melting, structure, guminess, melting in the mouth, the feeling left in the mouth, icy structure, lubricity, taste, cream taste, milk powder taste, foreign taste, vanilla taste and general acceptability added, is intended to determine. In the organoleptic evaluation, it was aimed to determine the effect of the added fat substitutes on the firmness, resistance to melting, structure, guminess, melting in mouth, feeling left in the mouth, icy structure, lubricity, taste, cream taste, milk powder taste, foreign taste, and vanilla taste.

\subsubsection{Statistical Analyses}

The research was analyzed by JMP statistical program for Windows developed by SAS (SAS JMP ${ }$ Statistical Discovery ${ }^{\mathrm{TM}}$ Version 7.0).

\section{Results and Discussion}

According to the results of variance analysis, the effect of fat level on the dry matter value of ice cream was statistically significant $(p<0.01)$, and fat $\times$ fat substitute interaction was very important $(p<0.01)$, but the effect of other factors was statistically insignificant $(p>0.05)$. The highest dry matter content (41.77\%) was determined on the 60th day of the storage in control group samples, while the lowest rate $(36.11 \%)$ was determined on the 60 th day of the storage in $2.5 \%$ fat with added Maltrin040 samples (Table 1). Although the amount of dry matter detected (41.77-36.11) was higher than the values reported by Keçeli [17] (29.33-31.54\%); it is similar to the results reported by Guinard et al. [18], Gönç et al. [19] and Ruger et al. [20]. According to the analysis of variance, the effect of fat level on the protein value of ice cream samples was statistically significant $(p<$ $0.01)$, and fat $\times$ fat substitute interaction was significant $(p<0.05)$; the effect of other factors was statistically insignificant $(p>0.05)$. The effect of fat substitute on the ash value was significant $(p<0.05)$, the effect of fat level and fat level $\times$ fat substitute interaction was significant $(p<0.01)$.

It was determined that the effect of fat substitutes addition on $\mathrm{pH}$ value of the ice cream was significant $(p<0.05)$, fat ratio was significant $(p<0.05)$, storage time and fat $\times$ fat substitute interaction were very important $(p<0.01)$. The $\mathrm{pH}$ values of almost all ice cream samples increased during the first 7 days of storage and then showed a downward trend (Table 1). The effect of fat, storage time and fat $\times$ fat substitute interaction was significant $(p<0.01)$ on the titration acidity (LA\%) (TA) of the experiment ice cream. The TA of the ice cream followed a fluctuating course until the end of the storage period. TA reached its highest value on the 90th day of the storage (Table 1). TA values in the study $(0.31 \%-0.45 \%)$ were higher than those reported by Gönç et al. [19] in vegetable fat ice cream $(0.25 \%-0.27 \%)$.

The effect of fat level on overrun was very significant $(p<0.01)$. As the fat content of ice cream decreased, the overrun decreased in parallel. The highest overrun value $(40 \%)$ was determined in control samples (Table 2). The overrun data for the other ice cream except the control group were lower than the values reported by Moraino and Alamprese [21] (35.7\%-50.9\%). On the other hand, Santos et al. [22] reported that the overrun values of ice cream samples produced with different mixes were minimum $22.45 \%$ and maximum 48.28\%. Botega et al. [23] reported this rate between $32 \%-73.1 \%$ in their studies.

The effect of fat level on the viscosity value of the product was significant $(p<0.05)$, while the effect of other factors was not significant $(p>0.05)$. According to the results from the study, the viscosity of $7.5 \%$ fat ice cream Maltrin040 or Simplesse $(100$ added was higher than the control group (Table 2). This situation was due to modified protein and starch. Similar studies using different fat substitutes have generally 
reported that low-fat ice creams have low viscosity. Aime et al. [24] reported that the viscosity of $2.4 \%$ and $0.5 \%$ fat ice cream produced using modified starch was much lower than $5 \%$ fat ice cream. Likewise, Li et al. [25] determined that the viscosity of $5.63 \%, 2.35 \%$ and $0.53 \%$ fat ice creams produced by using polydextrose based fat substitute Litesse ${ }^{\circledR}$ varies according to $9.65 \%$ fat control group. They reported that this change depends on fat and dry matter content. On the other hand, the amount of fat that should be used in the ice cream mixture varies between 3\%-16\% depending on the type of ice cream [26, 27]. However, Guinard et al. [18] reported that $14.30 \%-14.99 \%$ fat ice creams were most liked by the panelists.

An inverse proportion was found between the dry matter content of the ice cream samples and the first dripping time (Table 2). The reason for the shorter first dripping time of ice-creams with high solids content may be that the freezing point of the solution decreases as the amount of dissolved substance increases. The amount of non-fat dry matter determined in the ice-cream samples with low fat ratio was found to be proportionally higher than the others. This means that the amount of water-soluble solids of these ice creams was high. It has been reported by other researchers that solutions with a high amount of water-soluble solids dissolved faster [25]. The first dripping time was determined to be shorter in ice-creams with high fat content, i.e. the melting rate was high (Table 2). Roland et al. [18] reported that generally ice-cream with low fat content by adding various fat substitutes melts faster than the control group with a fat content of $10 \%$ and much slower than the control group with a fat content of $0.1 \%$.

It was calculated that fat ratio was very important $(p<0.01)$ and fat $\times$ fat substitute interaction was important $(p<0.05)$ on the organoleptic firmness value. It has been reported that ice-cream with low fat and dry matter ratio was firmer than higher ones [28]. The high fat content leads to the more fat aggregation and a softer product. The high rate of dry matter also allows the formation of proportionally less water crystals and reduces the firmness of the product. According to the results of the organoleptic research, contrary to expectations, ice cream with a low fat content (except $7.5 \%$ fat) was found to be statistically the same as the control group even though the firmness values were slightly lower. This situation shows that the fat substitutes used are effective on the formation of ice crystals. One reason for the low sensory firmness of ice cream samples with low fat content can be explained by the fact that the amount of fat droplets providing softness to the product in the colloidal phase is proportionally low, as well as the fat substitutes used to accumulate on the surface of the air bubbles to harden the product. Aime et al. [24] reported similar results in their modified starchy low fat ice cream.

It was found that the fat substitute was significant $(p<0.05)$, the fat ratio and the storage time were very significant $(p<0.01)$, and the fat substitute $\times$ storage time interaction was significant $(p<0.05)$ on the melting resistance values of the investigated ice cream samples. The melting resistance value of the samples Maltrin040 added (6.34) was higher than that of Simplesse ${ }^{\circledR} 100$ added (5.99) (Table 3). A quality ice cream sample is desirably resistant to melting at room temperature for a period of 10-15 min. Excessive addition of some emulsifying agents, as well as excessive melting delays, low dry matter content or coarse structure of ice-cream can cause very fast melting [28].

The effect of fat ratio was significant $(p<0.05)$ and the effect of fat $\times$ fat substitute interaction was very significant $(p<0.01)$ on the structure values of ice cream. The panelists scored both higher the structure of ice cream samples Simplesse ${ }^{\circledR} 100$ added and the control group. They also scored close to both groups. Ohmes et al. [29] reported that ice creams Simplesse added had a coarser structure compared to Dairy $\mathrm{Lo}^{\mathrm{TM}}$ added ice creams at the same rate. Roland et al. [14] 
Table 1 The values of some chemical and biochemical properties of the ice cream samples during 90 days storage period.

\begin{tabular}{|c|c|c|c|c|c|c|c|c|c|c|c|c|c|c|c|c|c|c|}
\hline \multirow{2}{*}{ Type of ice-cream } & \multicolumn{3}{|c|}{ Dry matter (\%) } & \multicolumn{3}{|c|}{ Fat in dry matter (\%) } & \multicolumn{3}{|c|}{ Protein $(\%)$} & \multicolumn{3}{|c|}{ Ash (\%) } & \multicolumn{3}{|c|}{ Titration acidity (LA\%) } & \multicolumn{3}{|c|}{$\mathrm{pH}$} \\
\hline & Min & Max & Mean & Min & Max & Mean & Min & Max & Mean & Min & Max & Mean & Min & Max & Mean & Min & Max & Mean \\
\hline $\begin{array}{l}\text { Simplesse } \AA 100+ \\
7.5 \% \text { fat }\end{array}$ & $\begin{array}{l}39.63 \\
(90 \mathrm{~d})\end{array}$ & $\begin{array}{l}39.74 \\
(60 . d)\end{array}$ & 39.63 & $\begin{array}{l}18.77 \\
(7 \mathrm{~d})\end{array}$ & $\begin{array}{l}19.17 \\
(30 \mathrm{~d})\end{array}$ & 18.92 & $\begin{array}{l}6.31 \\
(7 \mathrm{~d})\end{array}$ & $\begin{array}{l}6.38 \\
(1 \mathrm{~d})\end{array}$ & 6.34 & $\begin{array}{l}0.753 \\
(90 \mathrm{~d})\end{array}$ & $\begin{array}{l}0.755 \\
(30,60 \mathrm{~d})\end{array}$ & 0.754 & $\begin{array}{l}0.31 \\
(60 \mathrm{~d})\end{array}$ & $\begin{array}{l}0.35 \\
(90 \mathrm{~d})\end{array}$ & 0.34 & $\begin{array}{l}6.29 \\
(90 d)\end{array}$ & $\begin{array}{l}6.41 \\
(7 \mathrm{~d})\end{array}$ & 6.35 \\
\hline $\begin{array}{l}\text { Simplesse } \AA 100+ \\
5 \% \text { fat }\end{array}$ & $\begin{array}{l}37.16 \\
(60 \mathrm{~d})\end{array}$ & $\begin{array}{l}37.44 \\
(1 \mathrm{~d})\end{array}$ & 37.17 & $\begin{array}{l}13.46 \\
(1 \mathrm{~d})\end{array}$ & $\begin{array}{l}13.56 \\
(90 \mathrm{~d})\end{array}$ & 13.52 & $\begin{array}{l}6.75 \\
(15 \mathrm{~d})\end{array}$ & $\begin{array}{l}6.96 \\
(1 \mathrm{~d})\end{array}$ & 6.87 & $\begin{array}{l}0.706 \\
(15-90 d)\end{array}$ & $\begin{array}{l}0.711 \\
(1 \mathrm{~d})\end{array}$ & 0.707 & $\begin{array}{l}0.38 \\
(1,7 \mathrm{~d})\end{array}$ & $\begin{array}{l}0.42 \\
(90 d)\end{array}$ & 0.40 & $\begin{array}{l}6.23 \\
(60 \mathrm{~d})\end{array}$ & $\begin{array}{l}6.43 \\
(1 \mathrm{~d})\end{array}$ & 6.34 \\
\hline $\begin{array}{l}\text { Simplesse }{ }^{\circledR} 100+ \\
2.5 \% \text { fat }\end{array}$ & $\begin{array}{l}36.12 \\
(1,7 \mathrm{~d})\end{array}$ & $\begin{array}{l}38.58 \\
(30 \mathrm{~d})\end{array}$ & 36.13 & $\begin{array}{l}6.67 \\
(15 \mathrm{~d})\end{array}$ & $\begin{array}{l}7.10 \\
(60 \mathrm{~d})\end{array}$ & 6.88 & $\begin{array}{l}7.27 \\
(30 \mathrm{~d})\end{array}$ & $\begin{array}{l}7.80 \\
(60 \mathrm{~d})\end{array}$ & 7.60 & $\begin{array}{l}0.686(1, \\
7 \mathrm{~d})\end{array}$ & $\begin{array}{l}0.733 \\
(30 \mathrm{~d})\end{array}$ & 0.696 & $\begin{array}{l}0.42(1 \\
7,15 \mathrm{~d})\end{array}$ & $\begin{array}{l}0.45 \\
(90 \mathrm{~d})\end{array}$ & 0.43 & $\begin{array}{l}6.18 \\
(30 \mathrm{~d})\end{array}$ & $\begin{array}{l}6.33(1 \\
7 \mathrm{~d})\end{array}$ & 6.26 \\
\hline $\begin{array}{l}\text { Maltrin } 040+ \\
7.5 \% \text { fat }\end{array}$ & $\begin{array}{l}40.12 \\
(90 \mathrm{~d})\end{array}$ & $\begin{array}{l}41.06 \\
(60 \mathrm{~d})\end{array}$ & 40.12 & $\begin{array}{l}18.39 \\
(60 \mathrm{~d})\end{array}$ & $\begin{array}{l}18.83 \\
(90 \mathrm{~d})\end{array}$ & 18.65 & $\begin{array}{l}6.18 \\
(7 \mathrm{~d})\end{array}$ & $\begin{array}{l}6.24 \\
(1 \mathrm{~d})\end{array}$ & 6.20 & $\begin{array}{l}0.762 \\
(90 \mathrm{~d})\end{array}$ & $\begin{array}{l}0.780 \\
(60 \mathrm{~d})\end{array}$ & 0.770 & $\begin{array}{l}0.39 \\
(1,7,15 \\
\text { d) }\end{array}$ & $\begin{array}{l}0.42 \\
(90 \mathrm{~d})\end{array}$ & 0.40 & $\begin{array}{l}6.22 \\
(15 d)\end{array}$ & $\begin{array}{l}6.43 \\
(7 \mathrm{~d})\end{array}$ & 6.31 \\
\hline $\begin{array}{l}\text { Maltrin040+ } \\
5 \% \text { fat }\end{array}$ & $\begin{array}{l}37.07 \\
(15 \mathrm{~d})\end{array}$ & $\begin{array}{l}37.28 \\
(30 \mathrm{~d})\end{array}$ & 37.15 & $\begin{array}{l}13.67 \\
(90 \mathrm{~d})\end{array}$ & $\begin{array}{l}14.08 \\
(15 \mathrm{~d})\end{array}$ & 13.85 & $\begin{array}{l}6.63 \\
(15 \mathrm{~d})\end{array}$ & $\begin{array}{l}6.84 \\
(1 \mathrm{~d})\end{array}$ & 6.75 & $\begin{array}{l}0.704 \\
(15 \mathrm{~d})\end{array}$ & $\begin{array}{l}0.708 \\
(30 \mathrm{~d})\end{array}$ & 0.706 & $\begin{array}{l}0.38(1 \\
7,60 \mathrm{~d})\end{array}$ & $\begin{array}{l}0.39 \\
(90 d)\end{array}$ & 0.38 & $\begin{array}{l}6.29 \\
(30 \mathrm{~d})\end{array}$ & $\begin{array}{l}6.40 \\
(1,7 \mathrm{~d})\end{array}$ & 6.34 \\
\hline $\begin{array}{l}\text { Maltrin } 040+ \\
2.5 \% \text { fat }\end{array}$ & $\begin{array}{l}36.11 \\
(60 \mathrm{~d})\end{array}$ & $\begin{array}{l}36.14 \\
(1 \mathrm{~d})\end{array}$ & 36.13 & $\begin{array}{l}6.99 \\
(90 d)\end{array}$ & $\begin{array}{l}7.12 \\
(7 \mathrm{~d})\end{array}$ & 7.05 & $\begin{array}{l}7.35 \\
(30 d)\end{array}$ & $\begin{array}{l}7.89 \\
(60 \mathrm{~d})\end{array}$ & 7.69 & $\begin{array}{l}0.686(1, \\
7,15, \\
60,90 \mathrm{~d})\end{array}$ & $\begin{array}{l}0.687 \\
(30 \mathrm{~d})\end{array}$ & 686 & $\begin{array}{l}0.33 \\
(30 \mathrm{~d})\end{array}$ & $\begin{array}{l}0.40 \\
(90 \mathrm{~d})\end{array}$ & 0.38 & $\begin{array}{l}6.32 \\
(15 d)\end{array}$ & $\begin{array}{l}6.35 \\
(7 d)\end{array}$ & 6.33 \\
\hline $\begin{array}{l}\text { Control } \\
\text { (10\% fat) }\end{array}$ & $\begin{array}{l}41.67 \\
(7 d)\end{array}$ & $\begin{array}{l}41.77 \\
(60 d)\end{array}$ & 41.69 & $\begin{array}{l}23.98 \\
(60 \mathrm{~d})\end{array}$ & $\begin{array}{l}24.06 \\
(7 d)\end{array}$ & 24.02 & $\begin{array}{l}5.73 \\
(1,90 \mathrm{~d})\end{array}$ & $\begin{array}{l}5.78 \\
(7 \mathrm{~d})\end{array}$ & 5.75 & $\begin{array}{l}0.792(7 \\
15,90 \mathrm{~d})\end{array}$ & $\begin{array}{l}0.794 \\
(60 \mathrm{~d})\end{array}$ & .793 & $\begin{array}{l}0.37(1 \\
7,15 \mathrm{~d})\end{array}$ & $\begin{array}{l}0.41 \\
(90 d)\end{array}$ & 0.38 & $\begin{array}{l}6.29 \\
(90 d)\end{array}$ & $\begin{array}{l}6.41 \\
(7 \mathrm{~d})\end{array}$ & 6.35 \\
\hline
\end{tabular}

d: day; Min: minimum; Max: maximum.

Table 2 The values of some physical properties of the ice cream and mix samples.

\begin{tabular}{|c|c|c|c|c|c|}
\hline \multirow{2}{*}{ Type of ice cream } & \multirow{2}{*}{ Overrun (\%) } & \multirow{2}{*}{ Viscosity of mix $(\mathrm{cP})$} & \multirow{2}{*}{ First drip time (min) } & \multicolumn{2}{|c|}{ Melting rate $(\%)$} \\
\hline & & & & $30 \mathrm{~min}$ & $60 \mathrm{~min}$ \\
\hline Simplesse ${ }^{\circledR} 100+7.5 \%$ fat & 34.0 & 1,425 & 34.65 & 30.11 & 66.67 \\
\hline Simplesse ${ }^{\circledR} 100+5 \%$ fat & 32.5 & 1,300 & 25.30 & 40.02 & 80.45 \\
\hline Simplesse ${ }^{\circledR} 100+2.5 \%$ fat & 27.5 & 1,155 & 17.83 & 28.31 & 65.56 \\
\hline Maltrin $040+7.5 \%$ fat & 33.5 & 1,475 & 25.75 & 34.49 & 71.78 \\
\hline Maltrin $040+5 \%$ fat & 30.0 & 1,300 & 27.20 & 37.36 & 76.61 \\
\hline Maltrin $040+2.5 \%$ fat & 28.0 & 1,185 & 29.76 & 39.70 & 82.43 \\
\hline Control ( $10 \%$ fat) & 40.0 & 1,250 & 27.86 & 30.39 & 64.37 \\
\hline
\end{tabular}


Table 3 The values of some organoleptic properties of the ice cream samples during 90 days of storage.

\begin{tabular}{|c|c|c|c|c|c|c|c|c|c|c|c|c|c|c|c|}
\hline \multirow{2}{*}{ Type of ice cream } & \multicolumn{3}{|c|}{ Firmness } & \multicolumn{3}{|c|}{ Melting resistance } & \multicolumn{3}{|c|}{ Texture } & \multicolumn{3}{|c|}{ Guminess } & \multicolumn{3}{|c|}{ Melting in the mouth } \\
\hline & Min & Max & Mean & Min & Max & Mean & Min & Max & Mean & Min & Max & Mean & Min & Max & Mean \\
\hline $\begin{array}{l}\text { Simplesse }{ }^{\circledR} 100+ \\
7.5 \% \text { fat }\end{array}$ & $\begin{array}{l}6.00 \\
(30 \mathrm{~d})\end{array}$ & $\begin{array}{l}7.50 \\
(7 \mathrm{~d})\end{array}$ & 6.88 & $\begin{array}{l}5.64 \\
(90 \mathrm{~d})\end{array}$ & $\begin{array}{l}8.20 \\
(1 \mathrm{~d})\end{array}$ & 7.01 & $\begin{array}{l}5.13 \\
(30 \mathrm{~d})\end{array}$ & $\begin{array}{l}8.20(1,60, \\
90 \mathrm{~d})\end{array}$ & 7.56 & $\begin{array}{l}4.10 \\
(1 \mathrm{~d})\end{array}$ & $\begin{array}{l}7.69 \\
(30 \mathrm{~d})\end{array}$ & 5.34 & $\begin{array}{l}6.15 \\
(1 \mathrm{~d})\end{array}$ & $\begin{array}{l}7.69 \\
(90 \mathrm{~d})\end{array}$ & 6.88 \\
\hline $\begin{array}{l}\text { Simplesse }{ }^{\circledR} 100+ \\
5 \% \text { fat }\end{array}$ & $\begin{array}{l}6.00 \\
(7,30 \mathrm{~d})\end{array}$ & $\begin{array}{l}8.00 \\
(1 \mathrm{~d})\end{array}$ & 6.83 & $\begin{array}{l}5.13 \\
(90 \mathrm{~d})\end{array}$ & $\begin{array}{l}8.23 \\
(1 \mathrm{~d})\end{array}$ & 6.20 & $\begin{array}{l}5.64 \\
(7 \mathrm{~d})\end{array}$ & $\begin{array}{l}7.18 \\
(1 \mathrm{~d})\end{array}$ & 6.28 & $\begin{array}{l}3.08 \\
(7 \mathrm{~d})\end{array}$ & $\begin{array}{l}5.64 \\
(60 d)\end{array}$ & 4.28 & $\begin{array}{l}6.15 \\
(7 \mathrm{~d})\end{array}$ & $\begin{array}{l}7.18 \\
(1 \mathrm{~d})\end{array}$ & 6.67 \\
\hline $\begin{array}{l}\text { Simplesse }{ }^{\circledR} 100+ \\
2.5 \% \text { fat }\end{array}$ & $\begin{array}{l}4.50 \\
(1,15 \mathrm{~d})\end{array}$ & $\begin{array}{l}6.50 \\
(7 \mathrm{~d})\end{array}$ & 5.42 & $\begin{array}{l}5.13 \\
(30,90 \mathrm{~d})\end{array}$ & $\begin{array}{l}6.67 \\
(7 \mathrm{~d})\end{array}$ & 5.86 & $\begin{array}{l}5.13(30 \\
90 \mathrm{~d})\end{array}$ & $\begin{array}{l}7.69 \\
(7 d)\end{array}$ & 6.24 & $\begin{array}{l}3.08 \\
(1 \mathrm{~d})\end{array}$ & $\begin{array}{l}6.15(30 \\
90 \mathrm{~d})\end{array}$ & 4.92 & $\begin{array}{l}6.15(1,60 \\
\text { d) }\end{array}$ & $\begin{array}{l}6.67 \\
(7 \mathrm{~d})\end{array}$ & 6.28 \\
\hline $\begin{array}{l}\text { Maltrin } 040+ \\
7.5 \% \text { fat }\end{array}$ & $\begin{array}{l}5.50(7,15, \\
60 \mathrm{~d})\end{array}$ & $\begin{array}{l}6.50 \\
(30 \mathrm{~d})\end{array}$ & 5.79 & $\begin{array}{l}5.64 \\
(60 d)\end{array}$ & $6.67(7 \mathrm{~d})$ & 6.20 & $\begin{array}{l}5.13 \\
(1 \mathrm{~d})\end{array}$ & $\begin{array}{l}6.67 \\
(30,90 \mathrm{~d})\end{array}$ & 5.94 & $\begin{array}{l}3.08 \\
(1 \mathrm{~d})\end{array}$ & $\begin{array}{l}5.13 \\
(60 \mathrm{~d})\end{array}$ & 3.89 & $5.13(1 \mathrm{~d})$ & $\begin{array}{l}6.67 \\
(60 d)\end{array}$ & 5.99 \\
\hline $\begin{array}{l}\text { Maltrin } 040+5 \% \\
\text { fat }\end{array}$ & $\begin{array}{l}6.00 \\
\text { (D.w.s.) }\end{array}$ & $\begin{array}{l}6.00 \\
\text { (D.w.s.) }\end{array}$ & 6.00 & $\begin{array}{l}5.64 \\
(30,90 \mathrm{~d})\end{array}$ & $\begin{array}{l}6.15(1-15, \\
60 d)\end{array}$ & 5.98 & $\begin{array}{l}4.62(30 \\
90 \mathrm{~d})\end{array}$ & $\begin{array}{l}6.67 \\
(7 \mathrm{~d})\end{array}$ & 5.69 & $\begin{array}{l}2.56 \\
(7 \mathrm{~d})\end{array}$ & $\begin{array}{l}4.10(30 \\
90 \mathrm{~d})\end{array}$ & 3.29 & $\begin{array}{l}5.13(7, \\
30,90 \mathrm{~d})\end{array}$ & $\begin{array}{l}6.67 \\
(1 \mathrm{~d})\end{array}$ & 5.69 \\
\hline $\begin{array}{l}\text { Maltrin } 040+ \\
2.5 \% \text { fat }\end{array}$ & $\begin{array}{l}5.00 \\
(15 \mathrm{~d})\end{array}$ & $\begin{array}{l}6.50 \\
(60 \mathrm{~d})\end{array}$ & 5.92 & $\begin{array}{l}5.13(1,30, \\
90 \mathrm{~d})\end{array}$ & $\begin{array}{l}6.16 \\
(7 \mathrm{~d})\end{array}$ & 5.56 & $\begin{array}{l}5.64(30 \\
60,90 \mathrm{~d})\end{array}$ & $\begin{array}{l}6.67 \\
(7 \mathrm{~d})\end{array}$ & 6.03 & $\begin{array}{l}3.08 \\
(1 \mathrm{~d})\end{array}$ & $\begin{array}{l}4.10 \\
(7,30-90 \mathrm{~d})\end{array}$ & 3.85 & $\begin{array}{l}4.62 \\
(7 \mathrm{~d})\end{array}$ & $\begin{array}{l}6.15(1 \\
30,90 \mathrm{~d})\end{array}$ & 5.68 \\
\hline Control ( $10 \%$ fat) & $\begin{array}{l}6.00 \\
(1 \mathrm{~d})\end{array}$ & $\begin{array}{l}7.50 \\
(30,60 \mathrm{~d})\end{array}$ & 6.79 & $\begin{array}{l}5.64 \\
(30 \mathrm{~d})\end{array}$ & $\begin{array}{l}6.67 \\
(60 d)\end{array}$ & 6.28 & $\begin{array}{l}6.67(7,30, \\
60,90 \mathrm{~d})\end{array}$ & $\begin{array}{l}8.20 \\
(1 \mathrm{~d})\end{array}$ & 7.05 & $\begin{array}{l}4.10 \\
(1 \mathrm{~d})\end{array}$ & $\begin{array}{l}7.18 \\
(7 d)\end{array}$ & 5.22 & $\begin{array}{l}6.15 \\
(1 \mathrm{~d})\end{array}$ & $\begin{array}{l}7.18 \\
(60 d)\end{array}$ & 6.63 \\
\hline
\end{tabular}

1: worst score; 9: best score; d: day; D.w.s.: During the whole storage period; Min: minimum; Max: maximum.

Table 4 The values of some organoleptic properties of the ice cream samples during 90 days of storage (cont.).

\begin{tabular}{|c|c|c|c|c|c|c|c|c|c|c|c|c|c|c|c|}
\hline \multirow{2}{*}{ Type of ice cream } & \multicolumn{3}{|c|}{ Feeling left in the mouth } & \multicolumn{3}{|c|}{ Icy texture } & \multicolumn{3}{|c|}{ Lubricity } & \multicolumn{3}{|c|}{ Taste-aroma } & \multicolumn{3}{|c|}{ Cream taste } \\
\hline & Min & Max & Mean & Min & Max & Mean & Min & Max & Mean & Min & Max & Mean & Min & Max & Mean \\
\hline $\begin{array}{l}\text { Simplesse }{ }^{\circledR} 100+ \\
7.5 \% \text { fat }\end{array}$ & $\begin{array}{l}4.61 \\
(7 \mathrm{~d})\end{array}$ & $\begin{array}{l}7.69 \\
(30 \mathrm{~d})\end{array}$ & 6.37 & $\begin{array}{l}6.15 \\
(1 \mathrm{~d})\end{array}$ & $\begin{array}{l}7.69 \\
(90 \mathrm{~d})\end{array}$ & 7.01 & $\begin{array}{l}5.13 \\
(15 \mathrm{~d})\end{array}$ & $\begin{array}{l}7.69 \\
(60,90 \mathrm{~d})\end{array}$ & 6.58 & $\begin{array}{l}5.64 \\
(7 \mathrm{~d})\end{array}$ & $\begin{array}{l}7.18 \\
(1,90 \mathrm{~d})\end{array}$ & 6.63 & $\begin{array}{l}4.61 \\
(7 \mathrm{~d})\end{array}$ & $\begin{array}{l}7.69 \\
(30 d)\end{array}$ & 6.24 \\
\hline $\begin{array}{l}\text { Simplesse } \AA 100+ \\
5 \% \text { fat }\end{array}$ & $\begin{array}{l}5.13 \\
(30,90 \mathrm{~d})\end{array}$ & $\begin{array}{l}8.20 \\
(1 \mathrm{~d})\end{array}$ & 6.58 & $\begin{array}{l}6.15 \\
(60 d)\end{array}$ & $\begin{array}{l}6.67 \\
(1 \mathrm{~d})\end{array}$ & 6.29 & $\begin{array}{l}4.10 \\
(1 \mathrm{~d})\end{array}$ & $\begin{array}{l}6.67 \\
(7 \mathrm{~d})\end{array}$ & 5.90 & $\begin{array}{l}4.62 \\
(30 d)\end{array}$ & $\begin{array}{l}7.69 \\
(7 \mathrm{~d})\end{array}$ & 5.99 & $\begin{array}{l}5.13 \\
(90 \mathrm{~d})\end{array}$ & $\begin{array}{l}7.18 \\
(1 \mathrm{~d})\end{array}$ & 5.94 \\
\hline $\begin{array}{l}\text { Simplesse }{ }^{\circledR} 100+ \\
2.5 \% \text { fat }\end{array}$ & $\begin{array}{l}5.13 \\
(1 \mathrm{~d})\end{array}$ & $\begin{array}{l}7.18 \\
(7 \mathrm{~d})\end{array}$ & 5.90 & $\begin{array}{l}5.64 \\
(1 \mathrm{~d})\end{array}$ & $\begin{array}{l}7.18 \\
(7 \mathrm{~d})\end{array}$ & 6.54 & $\begin{array}{l}4.10 \\
(1 \mathrm{~d})\end{array}$ & $\begin{array}{l}6.67 \\
(7 \mathrm{~d})\end{array}$ & 5.51 & $\begin{array}{l}5.64 \\
(1,90 \mathrm{~d})\end{array}$ & $\begin{array}{l}7.69 \\
(7 d)\end{array}$ & 6.33 & $\begin{array}{l}4.10 \\
(1 \mathrm{~d})\end{array}$ & $\begin{array}{l}6.67 \\
(7 \mathrm{~d})\end{array}$ & 5.34 \\
\hline $\begin{array}{l}\text { Maltrin } 040+ \\
7.5 \% \text { fat }\end{array}$ & $\begin{array}{l}4.10 \\
(1 \mathrm{~d})\end{array}$ & $\begin{array}{l}5.64(30 \\
60,90 \mathrm{~d})\end{array}$ & 5.13 & $\begin{array}{l}4.62 \\
(30,90 d)\end{array}$ & $\begin{array}{l}7.18 \\
(1 \mathrm{~d})\end{array}$ & 5.69 & $\begin{array}{l}4.61 \\
(1 \mathrm{~d})\end{array}$ & $\begin{array}{l}6.16 \\
(60 d)\end{array}$ & 5.64 & $\begin{array}{l}6.15 \\
(7,90 \mathrm{~d})\end{array}$ & $\begin{array}{l}6.67 \\
(60 \mathrm{~d})\end{array}$ & 6.24 & $\begin{array}{l}4.10 \\
(1 \mathrm{~d})\end{array}$ & $\begin{array}{l}5.64 \\
(60 d)\end{array}$ & 4.83 \\
\hline $\begin{array}{l}\text { Maltrin } 040+5 \% \\
\text { fat }\end{array}$ & $\begin{array}{l}5.13 \\
(30,90 \mathrm{~d})\end{array}$ & $\begin{array}{l}6.15 \\
(1 \mathrm{~d})\end{array}$ & 5.60 & $\begin{array}{l}4.10 \\
(30,90 \mathrm{~d})\end{array}$ & $\begin{array}{l}7.18 \\
(1-15 \mathrm{~d})\end{array}$ & 5.98 & $\begin{array}{l}5.13 \\
(90 \mathrm{~d})\end{array}$ & $\begin{array}{l}6.15 \\
(1,60 \mathrm{~d})\end{array}$ & 5.77 & $\begin{array}{l}5.64(1,7, \\
15,90 \mathrm{~d})\end{array}$ & $\begin{array}{l}6.15 \\
(30,60 \mathrm{~d})\end{array}$ & 5.81 & $\begin{array}{l}4.10 \\
(1 \mathrm{~d})\end{array}$ & $\begin{array}{l}6.15 \\
(7 d)\end{array}$ & 5.13 \\
\hline $\begin{array}{l}\text { Maltrin } 040+ \\
2.5 \% \text { fat }\end{array}$ & $\begin{array}{l}5.13 \\
(30,90 \mathrm{~d})\end{array}$ & $\begin{array}{l}7.18 \\
(1,7,15 \mathrm{~d})\end{array}$ & 6.33 & $\begin{array}{l}4.10 \\
(30 \mathrm{~d})\end{array}$ & $\begin{array}{l}8.20 \\
(7 \mathrm{~d})\end{array}$ & 5.98 & $\begin{array}{l}4.10 \\
(1 \mathrm{~d})\end{array}$ & $\begin{array}{l}6.15 \\
(60 d)\end{array}$ & 5.04 & $\begin{array}{l}4.62(60 \\
90 \mathrm{~d})\end{array}$ & $\begin{array}{l}7.18 \\
(1 \mathrm{~d})\end{array}$ & 5.77 & $\begin{array}{l}4.10 \\
(1 \mathrm{~d})\end{array}$ & $\begin{array}{l}6.67 \\
(7,30 \mathrm{~d})\end{array}$ & 5.69 \\
\hline Control (10\% fat) & $\begin{array}{l}5.13 \\
(1 \mathrm{~d})\end{array}$ & $\begin{array}{l}7.18 \\
(30,60 \mathrm{~d})\end{array}$ & 6.16 & $\begin{array}{l}6.15 \\
(60,90 \mathrm{~d})\end{array}$ & $\begin{array}{l}8.20 \\
(7 \mathrm{~d})\end{array}$ & 7.09 & $\begin{array}{l}5.13 \\
(1 \mathrm{~d})\end{array}$ & $\begin{array}{l}6.15 \\
(7,60 d)\end{array}$ & 5.73 & $\begin{array}{l}7.69 \\
(7,60 d)\end{array}$ & $\begin{array}{l}8.20(1 \\
30,90 \mathrm{~d})\end{array}$ & 7.99 & $\begin{array}{l}7.18(1, \\
30,90 \mathrm{~d})\end{array}$ & $\begin{array}{l}7.69 \\
(7,60 \mathrm{~d})\end{array}$ & 7.39 \\
\hline
\end{tabular}

1: worst score; 9: best score; d: day; Min: minimum; Max: maximum. 
Table 5 The values of some organoleptic properties of the ice cream samples during 90 days of storage (cont. 2).

\begin{tabular}{|c|c|c|c|c|c|c|c|c|c|c|c|c|}
\hline \multirow{2}{*}{ Type of ice cream } & \multicolumn{3}{|c|}{ Milk powder taste } & \multicolumn{3}{|c|}{ Foreign taste } & \multicolumn{3}{|c|}{ Vanilla taste } & \multicolumn{3}{|c|}{ General acceptability } \\
\hline & Min & Max & 1 & in & Max & Mean & Min & Max & Mean & Iin & Max & Mean \\
\hline $\begin{array}{l}\text { Simplesse }{ }^{\circledR} 100+7.5 \% \\
\text { fat }\end{array}$ & $3.08(1,7,15 \mathrm{~d})$ & $\begin{array}{l}7.18(30,90 \\
\text { d) }\end{array}$ & 4.87 & $6.15(7 \mathrm{~d})$ & $8.20(30,90 \mathrm{~d})$ & 7.05 & $\begin{array}{l}6.15(30,90 \\
\text { d) }\end{array}$ & $\begin{array}{l}7.18(1,15,60 \\
\text { d) }\end{array}$ & 6.84 & $5.64(7 \mathrm{~d})$ & $20(1 \mathrm{~d})$ & 7.14 \\
\hline Simplesse ${ }^{\circledR} 100+5 \%$ fat & $\begin{array}{l}4.10(1,15,90 \\
\text { d) }\end{array}$ & $\begin{array}{l}4.62(30,60 \\
\text { d) }\end{array}$ & 4.27 & $6.15(7,60 \mathrm{~d})$ & $7.18(30,90 \mathrm{~d})$ & 6.62 & $4.62(30 \mathrm{~d})$ & $8.20(1 \mathrm{~d})$ & 6.62 & $\begin{array}{l}4.62(30,90 \\
\text { d) }\end{array}$ & 8.20 & 6.67 \\
\hline 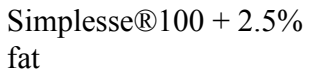 & $3.08(1 \mathrm{~d})$ & $7.18(60 \mathrm{~d})$ & 5.09 & $6.15(1 \mathrm{~d})$ & $7.69(7 \mathrm{~d})$ & 7.05 & $5.13(1 \mathrm{~d})$ & $8.20(7 \mathrm{~d})$ & 6.67 & $6.15(1,60 \mathrm{~d})$ & 7.69 & 6.54 \\
\hline Maltrin $040+7.5 \%$ fat & 3.08 & & .98 & 0.1 & $0 \mathrm{~d})$ & 7.26 & 4.1 & & 5.64 & & & 5.98 \\
\hline $40+5 \%$ fat & 3.08 & 7.1 & 4.23 & $5.64(7 \mathrm{~d})$ & 7.69 & 6.97 & $4.62(1,90 \mathrm{~d})$ & 7.18 & 5.43 & $5.64(7,90 \mathrm{~d})$ & 6.67 & 6.03 \\
\hline Maltrin $040+2.5 \%$ fat & $3.08(1 \mathrm{~d})$ & $6.67(7 \mathrm{~d})$ & 4.75 & $5.64(90 \mathrm{~d})$ & $8.20(1 \mathrm{~d})$ & 6.92 & $5.13(1 \mathrm{~d})$ & $8.20(7 \mathrm{~d})$ & 6.75 & $4.62(7 \mathrm{~d})$ & 7.18 & 5.94 \\
\hline Control (10\% fat) & $2.05(30 \mathrm{~d})$ & $7.18(60 \mathrm{~d})$ & 4.61 & $\begin{array}{l}6.67(30,90 \\
\text { d) }\end{array}$ & $\begin{array}{l}7.18(1,15,60 \\
\text { d) }\end{array}$ & 7.01 & $6.15(1 \mathrm{~d})$ & $8.20(60,90 \mathrm{~d})$ & 7.43 & $7.18(90 \mathrm{~d})$ & $\begin{array}{l}8.20(1,30,60 \\
\text { d) }\end{array}$ & 7.90 \\
\hline
\end{tabular}

1: worst score; 9: best score; d: day. 
reported that fat substitutes had a positive effect on the structure of the ice cream, but they could not provide the structural properties of $10 \%$ fat ice cream. According to the results of the study, although the sensory structure values of ice cream fat substitutes added were numerically low, they were generally similar to the control group.

It was determined that the effects of fat substitute, fat ratio and storage time were very important $(p<0.01)$ and fat $\times$ fat substitute interaction was significant $(p<$ $0.05)$ on the guminess values of the samples. Ice cream samples Maltrin040 added had the highest sensory guminess value (4.85), while Simplesse ${ }^{\circledR} 100$ added samples had the lowest (4.10) (Table 3). Ohmes et al. [29] also reported that Simplesse added ice creams have less gumy properties than those containing Dairy $\mathrm{Lo}^{\mathrm{TM}}$. The fact that the surface of the ice cream has a lean surface causes a gumy feeling [30]. The fact that the surface of the ice cream has a lean surface causes a gumy feeling [30].

The effects of fat $\times$ fat substitute and fat $\times$ storage time interaction were very significant $(p<0.01)$ on the feeling left in the mouth. Y1lsay et al. [31] compared the sensory and instrumental properties of low-fat $(6 \%)$ and non-fat $(0.5 \%)$ vanilla ice cream produced with milk protein-based fat substitute (Simplesse) with normal (12\% fat) and vanilla ice cream. They reported that ice creams containing $6 \%$ fat substitute did not show a significant difference, whereas the feeling left in the mouth levels of $0.5 \%$ fat ice creams was lower.

The effect of fat content and fat $\times$ fat substitute interaction on the icy structure value of the experiment ice creams was important $(p<0.05)$, while the effects of storage time and storage time $\times$ fat substitute interaction were very important $(p<0.01)$. Ohmes et al. [29] reported that the icy texture value of ice cream produced by using $5 \%$ Simplesse ${ }^{\circledR}$ instead of fat was 7.07 out of 15 . This value is below the values of the ice cream Simplesse ${ }^{\circledR} 100$ added in the current study (Table 4). It was reported that milk fat was not added to fat substitute in that study [29].

All the factors related to the lubricity values of ice cream samples were found to be insignificant $(p>0.05)$.

According to the results of variance analysis, the effect of fat ratio on the taste-aroma value of the samples was determined to be significant $(p<0.01)$. The highest sensory taste-aroma value was determined as 7.99 (control group) and the lowest taste-aroma value was 5.89 ( $5 \%$ fat ice cream) (Table 4 ).

It was determined that the effect of fat ratio and fat substitute $\times$ fat interaction on the cream taste value of ice cream samples was very significant $(p<0.01)$. The panelists scored the highest cream taste value to the control group ice creams and ice cream Simplesse ${ }^{\circledR} 100$ added (7.69) and the lowest cream taste value to Maltrin040 added samples (4.10) (Table 4). It is seen that the felt cream taste values are the same in the ice cream samples whose fat content is reduced in different proportions, but it is slightly higher than the control group. This demonstrates in particular that carbohydrate-based hydrated fat substitutes affect the melting of ice cream in the oral cavity and intensify the felt cream aroma [24].

It was shown that the effect of fat substitute was significant $(p<0.05)$, storage time was significant ( $p$ $<0.01)$ and fat $\times$ storage time interaction was significant $(p<0.01)$ on the taste of milk powder. It was found that all factors were insignificant on the foreign taste value of ice cream $(p>0.05)$.

According to the results of the variance analysis, it was seen that the fat substitute, fat ratio, storage time, fat substitute $\times$ fat interaction and storage time $\times$ fat $\times$ fat substitute interaction were very significant $(p<$ 0.01 ), moreover storage time $\times$ fat interaction and fat substitute $\times$ storage time interaction were significant $(p<0.05)$ on the vanilla taste value of the ice cream. It was recorded that the control group had the highest vanilla taste value (7.43), whereas ice cream Maltrin040 added had the lowest vanilla taste value (6.31) (Table 5). The vanilla flavor in the control group was more intense felt than the ice cream fat 
substitute added. This result is expected because the fat provides the transport of many flavor compounds. The fat interacts with said flavors to provide the flavor and aroma that no other fat substitute can produce. $\mathrm{Li}$ et al. [25] reported in their studies with HPLC (high performance liquid chromatography) that high-fat ice creams had more free vanillin than low-fat ones, but more time was needed to sensitize them. The researchers have explained this by stating that "high-fat ice cream melting in the mouth creates a more viscous layer on the tongue than low fat ones, thus preventing the taste of vanilla flavor". According to the study, it was determined that fat level of the ice cream was very important $(p<0.01)$, but other factors were insignificant $(p>0.05)$ on general acceptability of the experimental ice cream.

\section{Conclusion}

The decrease in viscosity as a result of fat reduction in ice cream production can be balanced by fat substitutes from protein and starch. Thus, the quality defect that will occur with the lack of fat will be eliminated. Also, for people with obesity problems, calorie-reduced ice creams will have been produced.

Panelists liked the Simplesse ${ }^{\circledR} 100$ added and control group ( $10 \%$ fat) ice creams in the first days of storage. In the last days of storage, especially the control group ice creams were preferred.

As a result of the findings, $7.5 \%$ fat ice creams $6 \%$ Simplesse ${ }^{\circledR} 100$ added can be recommended for ice creams to be produced by reducing fat ratio. In the near future, the production of ice-creams with fat substitutes at an industrial level will be of great importance for low-energy and vegetable fat dieters.

\section{Acknowledgement}

This study was produced titled "The Investigation of the Quality Features of Ice Cream Reduced Fat Rate Used by Some Fat Substitutes" supported by Harran University's Scientific Research Projects Coordinatorship (HUBAK Project No.: 708).

\section{References}

[1] Mohamed, A. A., Rayas-Duarte, P., Shogren, R. L., and Sessa, D. J. 2006. "Low Carbohydrates Bread, Formulation, Processing and Sensory Quality." Food Chem. 99: 686-92.

[2] Zahn, S., Forker, A., Krügel, L., and Rohm, H. 2013. "Combined Use of Rebaudioside A and Fibres for Partial Sucrose Replacement in Muffins." LWT-Food Sci. Tech. 50: 695-701.

[3] Akbari, M., Eskandari, M. H., and Davoudi, Z. 2019. "Application and Functions of Fat Replacers in Low-Fat Ice Cream: A Review." Trends in Food Science \& Technology 86: 34-40.

[4] Metin, M., and Koca, N. 1999. "Use of Fat Substitutes in Dairy Products." In Proceedings of the Food Science and Technology Congress in 2000s, pp. 63-8.

[5] Hamilton, M. P. 1990. "Ice Cream Manufacture." J. Soc. of Dairy Technol. 43 (1): 17-20.

[6] Zoulias, E. I., Oreopoulou, V., and Tzia, C. 2002. "Textural Properties of Low-Fat Cookies Containing Carbohydrate- or Protein-Based Fat Replacers." Journal of Food Engineering 55: 337-42.

[7] Lucca, P. A., and Tepper, B. J. 1994. "Fat Replacers and the Functionality of Fat in Foods." Trends in Food Science \& Technology 5: 12-9.

[8] Ognean, C. F., Darie, N., and Ognean, M. 2006. "Fat replacers-Review." Journal of Agroalimentary Processes and Technologies 12 (2): 433-42.

[9] Goff, H. D., and Hartel, R. W. 2013. Ice Cream. 7th ed. New York: Springer.

[10] Bradley, R. L., Arnold, J., Barbano, M., Semerad, R. G., Smith, D. E., and Vines, B. K. 1993. "Chemical and Physical Methods." In Standard Methods for the Examination of Dairy Products, 16th ed., edited by Marshall, R. T. Washington, DC: American Public Health Association, pp. 47-519.

[11] Kurt, A., Çakmakç1, S., and Çağlar, A. 1996. Guide to Milk and Products Inspection and Analysis Methods. Erzurum, Turkey: Atatürk University Faculty of Agriculture Publications.

[12] Gürsel, A., and Karacabey, A. 1998. "Calculations, Recipes and Quality Control Tests for Ice Cream Technology." Ankara University Faculty of Agriculture Publications, No. 1498, Deputy Textbook, Ankara, Turkey, 172.

[13] Cottrell, J. I. L., Pass, G., and Philips, G. O. 1979. "Assessment of Polysaccharides as Ice Cream Staibilizers.” J. Sci. Food Agric. 30: 1085-8.

[14] Roland, A. M., Philips, L. G., and Boor, K. J. 1999. "Effect of Fat Content on the Sensory Properties, Color, Melting and Hardness of Ice Cream." J. Dairy Sci. 82: $32-8$. 
[15] Chung, S. J., Heymann, H., and Grun, I. U. 2003. "Application of GPA and PLSR in Correlating Sensory and Chemical Data Sets." Food Quality and Preference 14: 485-95.

[16] Atsan, E. 2004. "Effect of Emulsifiers and Stabilizers Used on Different Proportions on Some Quality Criteria of Ice-Cream.” PhD thesis, Atatürk University Science Institute.

[17] Keçeli, T. 1995. "A Comparative Study on the Effects of Different Stabilizers on Some Properties of Ice Cream Made from Cow and Goat Milk." Master thesis, Çukurova University Science Institute.

[18] Guinard, J. X., Zoumas-Morse, C., Mori, L., Uatoni, B., Panyam, D., and Kilara, A. 1996. "Effect of Sugar and Fat on the Acceptability of Vanilla Ice Cream." J. Dairy Sci. 79: 1922-7.

[19] Gönç, S., Oktar, B., and Enfiyeci, A. S. 1988. "A Research on Possibilities of Using Vegetable Fat Instead of Milk Fat in Ice Cream." Ege University Faculty of Agriculture Journal 25 (1): 10-22.

[20] Ruger, P. R., Baer, R. J., and Kasperson, K. M. 2002. "Effect of Double Homogenization and Whey Protein Concentrate on the Texture of Ice Cream." J. Dairy Sci. 85: 1684-92.

[21] Moriano, M. E., and Alamprese, C. 2017. "Honey, Trehalose and Erythritol as Sucrose-Alternative Sweeteners for Artisanal Ice Cream: A Pilot Study." LWT-Food Science and Technology, pp. 329-34.

[22] Santos, G. G., and Silva, M. R. 2012. "Mangaba (Hancornia speciosa Gomez) Ice Cream Prepared with Fat Replacers and Sugar Substitutes." Ciência $e$ Tecnologia de Alimentos 32 (3): 621-8.
[23] Botega, D. C. Z., Marangoni, A. G., Smith, A. K., and Goff, H. D. 2013. "The Potential Application of Rice Bran Wax Oleogel to Replace Solid Fat and Enhance Unsaturated Fat Content in Ice Cream.” Journal of Food Science 78 (9): 1334-9.

[24] Aime, D. B., Arntfield, S. D., Malcolmson, L. J., and Ryland, D. 2001. "Textural Analysis of Fat Reduced Vanila Ice Cream Products." Food Research International 34 (2): 237-46.

[25] Li, Z., Marshall, R., Heymann, H., and Fernando, L. 1997. "Effect of Milk Fat Content on Flavor Perception of Vanilla Ice Cream.” J Dairy Sci. 80: 3133-41.

[26] Adapa, S., Dingeldein, H., Schmidt, K. A., and Herald, T. J. 2000. "Rheological Properties of Ice Cream Mixes and Frozen Ice Creams Containing Fat and Replacers." $J$. Dairy Sci. 83: 2224-9.

[27] Tekinşen, O. C. 2000. Dairy Products Technology, 3rd ed. Konya: Selçuk University Printing House, 329.

[28] Bodyfelt, M. S., Tobias, J., and Trout, G. M. 1988. The Sensory Evaluation of Dairy Products. Van Nostrad Reinhold, 598.

[29] Ohmes, R. L., Marshall, R. T., and Heymann, H. 1998. "Sensory and Physical Properties of Ice Creams Containing Milk Fat or Fat Replacers.” J Dairy Sci. 81: 1222-8.

[30] Pentince, J. H. 1992. Dairy Rheology: A Concise Guide. New York: VCH Publishers Inc., 163.

[31] Yılsay, T. Ö., Y1lmaz, L., and Bayızit, A. A. 2005. “The Effect of Using a Whey Protein Fat Replacer on Textural and Sensory Characteristics of Low-Fat Vanilla Ice Cream." European Food Research and Technology 222 (1): $171-5$. 